\title{
BMJ Open Patient and nurse preferences for nurse handover-using preferences to inform policy: a discrete choice experiment protocol
}

\author{
Jean Spinks, ${ }^{1}$ Wendy Chaboyer, ${ }^{2}$ Tracey Bucknall, ${ }^{3}$ Georgia Tobiano, ${ }^{4}$ \\ Jennifer A Whitty ${ }^{5}$
}

To cite: Spinks J, Chaboyer W, Bucknall T, et al. Patient and nurse preferences for nurse handover-using preferences to inform policy: a discrete choice experiment protocol. BMJ Open 2015;5:e008941. doi:10.1136/bmjopen-2015008941

- Prepublication history for this paper is available online. To view these files please visit the journal online (http://dx.doi.org/10.1136/ bmjopen-2015-008941).

Received 30 May 2015 Revised 23 August 2015 Accepted 9 October 2015

CrossMark

For numbered affiliations see end of article.

Correspondence to

Dr Jean Spinks;

j.spinks@griffith.edu.au

\begin{abstract}
Introduction: Nursing bedside handover in hospital has been identified as an opportunity to involve patients and promote patient-centred care. It is important to consider the preferences of both patients and nurses when implementing bedside handover to maximise the successful uptake of this policy. We outline a study which aims to (1) identify, compare and contrast the preferences for various aspects of handover common to nurses and patients while accounting for other factors, such as the time constraints of nurses that may influence these preferences.; (2) identify opportunities for nurses to better involve patients in bedside handover and (3) identify patient and nurse preferences that may challenge the full implementation of bedside handover in the acute medical setting.
\end{abstract}

Methods and analysis: We outline the protocol for a discrete choice experiment (DCE) which uses a survey design common to both patients and nurses. We describe the qualitative and pilot work undertaken to design the DCE. We use a D-efficient design which is informed by prior coefficients collected during the pilot phase. We also discuss the face-to-face administration of this survey in a population of acutely unwell, hospitalised patients and describe how data collection challenges have been informed by our pilot phase. Mixed multinomial logit regression analysis will be used to estimate the final results.

Ethics and dissemination: This study has been approved by a university ethics committee as well as two participating hospital ethics committees. Results will be used within a knowledge translation framework to inform any strategies that can be used by nursing staff to improve the uptake of bedside handover. Results will also be disseminated via peer-reviewed journal articles and will be presented at national and international conferences.

\section{INTRODUCTION}

Clinical handover is an important aspect of safe patient care. ${ }^{1}$ The definition of clinical handover is 'the transfer of professional responsibility and accountability for some or

\section{Strengths and limitations of this study}

- This study uses a unique discrete choice experiment design to elicit the preferences of patients and nurses for the most important aspects of bedside handover common to both groups.

- Results will be used within a knowledge translation framework to identify any barriers to full implementation of bedside handover and to develop specific strategies to overcome them and increase the likelihood of uptake.

- The survey will be conducted in Australia in an acute care, tertiary setting and results may not be generalisable to other settings.

all aspects of care for a patient, or groups of patients, to another person or professional group on a temporary or permanent basis'. Shift-to-shift handover between nurses normally occurs 2-3 times/day in most hospitals and is an opportunity to promote a patientcentred approach to care..$^{3-6}$

The Australian Commission on Safety and Quality in Healthcare ${ }^{7}$ advocates bedside handover with active patient participation. While this guideline recognises that handover practices must be adapted to specific clinical environments, it recommends against handover being undertaken in a common staff area. ${ }^{7}$ Handovers should occur in places that allow patients the chance to hear what is being said, correct any misinformation and ask questions about their care. ${ }^{8}$ While there is evidence that in general, nurses support patient participation, ${ }^{5}$ nurses control the physical location for bedside handover, not always conducting handover at the bedside, instead standing outside the patient's room or at the nurses' station ${ }^{9}$ which may hinder patient participation.

Given the impetus to promote handover at the bedside, it is important to understand 
both enablers and barriers to bedside handover, as perceived by nurses and patients. Previous literature reviews on handover are abundant ${ }^{10-13}$ (see also the online appendix in Staggers and Blatz ${ }^{12}$ for a full list); however, only one systematic review has focused on nursing bedside handover. ${ }^{10}$ In this review, Anderson $e t a l^{10}$ analysed 45 articles to better understand barriers to the implementation of bedside handover. Of the seven key issues identified by the authors, three are most relevant to a discussion of preferences for handover including (1) confidentiality; (2) time perceptions of bedside handover and (3) the degree of patient/carer involvement.

\section{Nurses' perceptions of bedside handover issues}

Anderson et al's key issues are supported by other evidence exploring nurse perceptions of handover. ${ }^{5} 1012$ 14-27 In terms of the issue of confidentiality, mixed results are found, with some nurses voicing concerns around privacy and confidentiality when handing over information at the bedside. ${ }^{22}$ However, other nurses believe that this issue is manageable, through more discrete strategies. ${ }^{17} 2425$ Another key issue in relation to bedside handover is the perceived time it takes to perform a bedside rather than recorded handover. Previous researchers have demonstrated that nurses felt pressured and lacked time to conduct bedside handovers. $^{9} 27$ However, contrasting views were shown by Anderson and Mangino ${ }^{23}$ who found nurses' perceived bedside handover to be less time-consuming compared to Jeffs $e t a l^{26}$ who found it to be more time-consuming, despite receiving more succinct and efficient information. Wilson ${ }^{21}$ found variable views among participants. This is likely an important factor which shapes nurses preferences for bedside handover given their already significant workload. The third issue relevant here is the preference for the level of patient and/or carer involvement in handover. Nurses valued bedside handover as it offered a way for patients to participate ${ }^{52}$ by intercepting errors and clarifying plans and informations, ${ }^{26}$ and believed that their patients were actively involved in the bedside handover process. $^{28}$ However, other nurses prefer little patient engagement in handover, viewing the patient as a source of disruption, ${ }^{27}$ sometimes standing in the doorway to curb patient involvement. ${ }^{24}$ Observations of bedside handovers support the latter, with nurse-patient interaction not occurring in all cases. $^{29}$

\section{Patients' perceptions of bedside handover issues}

There is relatively less literature in relation to patient perceptions of bedside handover, ${ }^{4} 6825$ 30-33 however this is increasing. Of the three key themes discussed above for nurses, only two, confidentiality and the level of involvement are relevant to patients. The evidence around perceptions of confidentiality in relation to bedside handover is mixed. Greaves ${ }^{33}$ found evidence that confidentiality was an issue for some patients when handover was conducted at the bedside while McMurray found that patients were less concerned about this issue. ${ }^{4}$ Although some patients have expressed that they do not have issues with their information being handed over at the bedside, they do believe certain topics should be handled with discretion. ${ }^{31}{ }^{34}$ In relation to the level of involvement in handover, evidence to date suggests that patients are not routinely involved..$^{5} 8$ In fact, patients appear to have varying views for the level of participation they want to undertake. ${ }^{15} 3134$ Despite this, a number of studies reported that patients feel more involved and it is beneficial to their care if they are actively involved in the handover process. ${ }^{4212333}$

Overall, there is a body of evidence that addresses patients' and nurses' perceived issues with bedside handover. Our study will compliment these findings by addressing patients' and nurses' preferences for each of these perceived issues. This will provide a measure of the relative strength and ordering of preferences in relation to different issues of bedside handover. Given that effective bedside handover involves both patients and nurses and that the preferences of both groups will affect the implementation of the recommended guidelines, evidence is needed to assess both parties preferences. It is also important to identify any apparent disconnect in the preferences of both groups. We outline a unique study protocol designed to investigate patient and nurse preferences for bedside handover. We detail a discrete choice experiment (DCE) methodology which allows for patient and nurse preferences to be captured using the same survey, with slight modifications, so that both similarities and differences can be identified between the groups. This information will be used to inform the ongoing implementation of bedside handover in a way that is beneficial to both patients and nurses.

\section{AIIMS}

We have identified three main objectives for this study, namely:

1. To identify, compare and contrast the preferences for various aspects of handover common to nurses and patients while accounting for other factors, such as the time constraints of nurses that may influence these preferences. The aspects of handover to be explored include whether the patient is invited to participate; the number of nurses involved in handover; if a family member is allowed to be present; the level of patient involvement; what information is discussed at handover; and how confidential information is exchanged. The impact of other factors on preferences such as individual characteristics, the number of hospitalisations (patients) and workload constraints (nurses) will be accounted for by their inclusion in the analysis.

2. To identify opportunities for nurses to better involve patients in bedside handover. 
3. To identify patient and nurses preferences that may challenge the full implementation of bedside handover in the acute medical setting.

\section{METHODS AND ANALYSIS}

\section{Overview of approach to DCE design}

A DCE is a type of survey that has been increasingly used to assess preferences for health and healthcare..$^{35} 36$ It asks participants to imagine particular scenarios, designed to mimic 'real life', and make a choice based on their own preferences between different alternatives in each scenario. DCE methodology allows researchers to estimate the relative strength of preferences for particular attributes in quantitative terms. The dependent variable in the model represents the likelihood of choosing a particular bundle of attributes, known as an 'alternative', over any other alternative in a given group. The independent variables are the levels of the particular attributes included in the survey as seen by participants. Interpersonal differences can be accounted for by their inclusion as covariates and/or their specification in a mixed logit model to explain the extent to which individual preferences vary around the mean sample preference parameter. Given the aim to identify both similarities and differences between patient and nurse preferences, we designed a DCE survey that can be presented to both patients and nurses with only slight wording modifications, to support a direct comparison of preferences.

\section{Rationale for using a DCE}

DCE methodology has been influenced by psychology and economics and is used in fields as diverse as marketing, environmental economics and psychology. ${ }^{37} 38$ The basic premise is that satisfaction, or utility, is derived from the component parts of a good or service, which are known as attributes. The levels of different attributes are varied and presented together within a simulated choice and survey participants are asked to choose which alternative they would prefer from the options presented. It is important that the choice set includes all possible alternatives, which may include an opt out alternative. The choice data are analysed using regression modelling in a random utility framework, in which participants are assumed to choose the alternative which maximises their overall utility. By asking participants to make repeated choices, statistical precision is increased.

DCEs provide a better understanding of preferences than instruments such as satisfaction surveys as the relative ranking of improvements in different attributes can be achieved. DCE methodology is complementary to qualitative analysis which allows a deeper understanding of why and how preferences have been formed. We will use DCE methodology in this study to directly compare the relative strength of preferences for different aspects of bedside handover in quantitative terms, which cannot be undertaken with qualitative analysis. The relative rankings of preferences will provide insight for clinical leaders and policy makers to identify which aspects of bedside handover are most important to which groups and can guide priority setting for the implementation of changes that support bedside handover as a common practice. For example, if it is found that patients, in general and accounting for a range of interpersonal differences, strongly prefer to be invited to participate in handover rather than not, then this provides evidence to clinical leaders that an invitation to participate should be considered for inclusion in practice guidelines.

The DCE was developed according to best practice guidelines. ${ }^{39}$ It is recognised that qualitative work is required as part of DCE development to identify the attributes and levels relevant to the choice and as part of the pilot testing of the DCE survey. ${ }^{40}$

\section{Qualitative study to inform development of the DCE}

This study was informed by qualitative work which was undertaken prior to the development of the attributes and levels. ${ }^{41}{ }^{42}$ A literature review ${ }^{6}$ was conducted which identified barriers and facilitators to patient participation in nursing care. In particular, information-sharing encounters, such as bedside handover, were seen to improve patient participation. Next, the qualitative study was conducted at two different hospitals, one public and one private, in two different states of Australia. Twenty medical patients and 20 nurses were interviewed between November 2013 and March 2014 to elicit their preferences for patient participation. Registered and enrolled (similar to practical) nurses were both invited to participate, in line with the proposed sample for the DCE. Semistructured interviews were undertaken and audio-taped. Patients and nurses were asked their perceptions and experiences of patient participation in the bedside handover. Importantly, if they saw no role for patients in bedside handover, they were probed further to find out why this was their preference. In addition, the participants were asked more general questions about patient participation including what it meant to them, their role and the barriers and facilitators to it, which is reported elsewhere. ${ }^{41}{ }^{42}$ Patient and nurse interviews were transcribed verbatim and analysed separately using content analysis.

In terms of bedside handover, we found that some nurses approved patients' role in handover, as they believed they could provide updated information and clarify errors and valued the expert knowledge patients could share. ${ }^{42}$ Nurses stated that they played a role in encouraging patients' participation by introducing themselves and informing the patient. However, not all nurses shared this view; some nurses did not encourage patient participation in bedside handover as they believed patients interfered or they felt they had to restrict information and keep it confidential. For patients, they expressed motivation to participate in their care. ${ }^{41}$ Patients viewed bedside handover as an opportunity to gain information, which gave them the confidence to 
share information during this exchange. ${ }^{41}$ However, patients' confidence to participate was diminished if the nurse did not display a manner that invited them to participate. ${ }^{41}$ Based on these findings, attributes were identified for consideration in the DCE survey.

\section{Selection of attributes and levels for the DCE}

A consensus group, comprising of nurses researching in the area of handover and patient participation, health economists and a health advocate who has worked in the area of handover was formed to synthesise the attributes and level for the DCE survey. The final list of attributes was arrived at following guidance from Bridges et $a l^{39}$ by (1) considering any possible attribute thought to be relevant to the decision to prefer a particular handover situation compared to another (based on the literature review and previous qualitative work $^{41}{ }^{42}$ ); (2) including those who could be realistically described in choice scenarios and were potentially amenable to change and (3) holding constant any attributes that were considered relevant for realism, but that could not be included (namely, the time of day handover occurred). All attributes that met these criteria were included in the DCE design. Six attributes were considered to be relevant-(1) whether the patient is explicitly invited to participate in the handover process; (2) the number of nurses present at the handover; (3) if a family member or trusted friend is allowed by the nursing staff to be present; (4) the level of involvement of the patient, that is, whether participation is passive, active or somewhere in between; (5) whether the information discussed at handover is limited to clinical information only, or if it includes a plan for care; and (6) the level of confidentiality and privacy with which sensitive information is discussed. This list formed the basis of the DCE design, which is presented in table 1 for patients and in table 2 for nurses.

A consumer health advocate was engaged once consensus was reached to revise the wording in plain
English. Given that nursing handover occurs between shifts, on average, around two to three times a day in most hospitals, it was decided to ask patients and nurses to imagine the handover that occurs just after lunch (around 13:00 or 14:00) when responding to the survey. This time was chosen as patients are most likely to be more alert at this time of day (rather than early morning or late at night when other shift changes occur).

\section{DCE design}

As detailed in Hensher et $a l_{,}^{43}$ the number of possible unique choice profiles, given the inclusion of the specified number of attributes and levels is $\mathrm{L}^{\mathrm{A}}$ in an unlabelled DCE, where $\mathrm{L}$ is the number of levels and A the number of attributes. Thus, the combination of attributes and levels shown in table 1 results in a possible $2^{4} \times 3^{2}=144$ alternative handover profiles. While this is not an impossibly large number of profiles to consider, we were cognisant of previous DCE work undertaken in a group of very sick patients ${ }^{44}$ and recognised that we needed to limit the number of choice sets presented to patients so as not to overburden them. For this reason, it was decided to pilot test a maximum of six choice sets per patient participant. We decided to pilot nine choice sets per nurse participant as although nurses are likely to have time constraints when answering the survey (on shift), they are well practised at assessing complex information quickly. However, both the patient and nurse surveys used the same overall DCE design so that results for the two groups could be easily compared without considering that any survey bias is likely to influence the groups in different ways.

A D-efficient experimental design was used to maximise the efficiency (precision) of results in the main survey. ${ }^{45}$ In the first instance we estimated a D-efficient multinomial logit (MNL) fractional factorial main effects design, generating 36 choice sets using Ngene software. ${ }^{46}$ Owing to the time and fatigue constraints of

Table 1 Attributes and levels for patient survey

\begin{tabular}{|c|c|c|}
\hline Attributes & Levels for handover $A / B$ at bedside & Levels for 'handover elsewhere' \\
\hline I am invited to participate & Yes (1), no (2) & No \\
\hline $\begin{array}{l}\text { Number of nurses present at the } \\
\text { handover }\end{array}$ & $\begin{array}{l}\text { Only the nurse leaving and the nurse coming on (1); } \\
\text { the nursing team leaving and the team coming on (2) }\end{array}$ & \\
\hline $\begin{array}{l}\text { Family member, carer or trusted } \\
\text { friend allowed to be present }\end{array}$ & Yes (1), no (2) & No \\
\hline Level of involvement & $\begin{array}{l}\text { I hear what is said ( } 3 \text { ); I hear what is said and I am } \\
\text { asked questions (2); I hear what is said, I am asked } \\
\text { questions and I can speak up at any time (1) }\end{array}$ & None (hybrid base) \\
\hline $\begin{array}{l}\text { What information related to your } \\
\text { care is discussed }\end{array}$ & $\begin{array}{l}\text { Information about my medical condition only (2); } \\
\text { Information about my medical condition and plan for } \\
\text { care (1) }\end{array}$ & Unknown (hybrid base) \\
\hline Confidentiality and privacy & $\begin{array}{l}\text { Sensitive information is handed over quietly at my } \\
\text { bedside (3); sensitive information is handed over } \\
\text { verbally away from my bedside (2); sensitive } \\
\text { information is handed over in written form (1) }\end{array}$ & $\begin{array}{l}\text { Likely to take place in a nurses } \\
\text { station, tea-room or meeting room } \\
\text { (hybrid base) }\end{array}$ \\
\hline
\end{tabular}

Levels for 'handover elsewhere'

I am invited to participate

Number of nurses present at the

handover

Family member, carer or trusted

friend allowed to be present

Level of involvement

What information related to your care is discussed

Confidentiality and privacy 
Table 2 Attributes and levels for nurse survey

\begin{tabular}{|c|c|}
\hline Attributes & Levels \\
\hline The patient is invited to participate & Yes (1), no (2) \\
\hline Number of nurses present at the handover & $\begin{array}{l}\text { Only the nurse leaving and the nurse coming on (1); the nursing team leaving } \\
\text { and the team coming on (2) }\end{array}$ \\
\hline $\begin{array}{l}\text { Family member, carer or trusted friend of } \\
\text { the patient allowed to be present }\end{array}$ & Yes (1), no (1) \\
\hline Level of patient involvement & $\begin{array}{l}\text { The patient can hear what is said (3); the patient can hear what is said and is } \\
\text { asked questions (2); the patient can hear what is said, is asked questions and } \\
\text { can speak up at any time (1) }\end{array}$ \\
\hline $\begin{array}{l}\text { What information related to patient care is } \\
\text { discussed }\end{array}$ & $\begin{array}{l}\text { Information about the patient's medical condition only (2); information about the } \\
\text { patient's medical condition and plan for care (1) }\end{array}$ \\
\hline Confidentiality and privacy & $\begin{array}{l}\text { Sensitive information is handed over quietly at the bedside (3); sensitive } \\
\text { information is handed over verbally away from the bedside (2); sensitive } \\
\text { information is handed over in written form (1) }\end{array}$ \\
\hline
\end{tabular}

respondents considered above, only a subset of the full design will be shown to each participant. Thus, the full design was separated (blocked) into six versions of the survey for patients (6 choice sets per participant) and four versions of the survey for nurses (9 choice sets per participant). Blocking is a statistical technique which is accepted practice in DCE design ${ }^{43}$ to create subsets of the full design. The survey will be administered on an iPad, and the version (block) of the survey will be randomly allocated to each participant by the software used to administer the survey; however, the choice sets within blocks will not be randomised. Pilot data were collected on 20 patients and 10 nurses. These data were used to estimate a multinomial (MNL) choice $\operatorname{model}^{43}$ to produce separate prior coefficients for patients and nurses. Prior information collected in the pilot DCE was used to inform the direction and magnitude of the likely coefficients in the final model. To account for the different coefficients for both patients and nurses, a 'model-averaging' approach ${ }^{47}$ was used to develop the final design. This means estimating the same design for both groups using their respective priors, but weighting them differently given their likely influence in the final sample. Given that our predicted sample size for patients $(\mathrm{N}=400)$ was double that for nurses $(\mathrm{N}=200)$, it was decided to double-weight the patient model. We also allowed for uncertainty in the prior estimates by taking a Bayesian approach ${ }^{48}$ whereby a range of coefficients were considered likely for the less certain attribute estimates. 1000 Guass draws were used to simulate the distributions from which the Bayesian parameters were drawn. Although complete attribute balance was not possible for this design, it was forced to be as high as possible without decreasing the efficiency of the design.

\section{Pilot of DCE}

The DCE was piloted in 20 patients and 10 nurses recruited from a ward not participating in the main study in October and November 2014. It took on an average $22 \mathrm{~min}$ for patients and $11 \mathrm{~min}$ for nurses to complete the survey. Participants were asked at the end of the survey what they thought could be improved about the survey. A multinomial choice model was estimated using the pilot data to confirm the face validity of the survey instrument.

Undertaking the pilot highlighted important procedural issues for the main survey data collection. Most participants enjoyed undertaking the survey on an iPad, however we found it important to assess participants' iPad competence by questioning them prior to administration of the survey, allowing us to cater our assistance as required. In the hospital environment, we identified infection control as an important issue when using an iPad, and used disinfectant wipes to clean the iPad between users. In terms of recruitment, patient turnover needs to be considered. We found less eligible and willing participants when recruiting on consecutive days, therefore non-consecutive days were preferred whenever possible. For nurses, recruitment can be challenging due to their busy workloads. To account for this, we checked that no events, such as education sessions, were occurring on the ward and surveyed nurses after the afternoon handover when there was both morning and afternoon nurses present.

\section{Sample selection}

The DCE survey will be administered to patients and nurses in medical wards of two metropolitan hospitals in different states of Australia-one publically and one privately funded. The public hospital is located in Queensland and is a metropolitan tertiary referral hospital with 750 beds. The private hospital is located in Victoria and is a metropolitan tertiary referral hospital with 508 beds.

Sample size calculations for DCEs are currently an emerging field of enquiry. ${ }^{49}$ A number of 'rules of thumb' exist in the literature and we used guidance from Johnson and Orme ${ }^{4950}$ to estimate that we would require a minimum sample size of 125 patients and 83 nurses to give precise estimates of main effects. However, we chose to recruit a more generous sample size to 
allow for the inclusion of individual characteristics as covariates in the model.

Patients will be considered eligible to participate (both pilot and main survey) if they are an adult medical patient (age $\geq 18$ years); currently an inpatient on one of the participating wards; have sufficient English language skills to participate; able and willing to provide informed consent; and have been in hospital as an inpatient for at least 2 days before recruitment (so they have some experience of being a patient in hospital). To decrease patient burden, nursing shift managers will first assess if a patient is considered to be eligible and ask if they are willing to be approached by a researcher with more information. The nurse research assistants will then approach each patient who agrees, provide an information sheet, confirm eligibility, consent the patient and undertake the survey.

Registered and enrolled (similar to practical) nurses working in the same medical ward as the patients recruited are eligible to participate. No pool or agency nurses will be recruited. Potential participants will be approached by the nursing shift manager or their designate to be asked if they would like to hear about the research before being approached. If a nurse agrees, a written consent form will be completed and the nurse will be interviewed on that day.

Records will be kept of the number of eligible patients and nurses who decline to participate to provide an overall response rate.

\section{Survey administration}

Given the practical difficulties of asking very sick patients to complete a survey, it was decided to employ nurse research assistants to collect the survey data in person using electronic tablets (iPads). iPads are small and relatively light and can be easily moved around by a patient to find a comfortable position. Each choice set will appear on a separate screen. An example choice set is shown in figure 1. Data on demographics (age, gender, country of birth) will be collected for all participants. In addition, data on patients' clinical condition such as current pain level, perceived health and length of stay will be collected. Specific data on nurses such as years of experience, supervisory responsibilities and workload will be obtained (table 3 ).

\section{Data analysis plan}

Initially, a MNL model will be used to estimate average preferences for patients and nurses separately. The MNL model is the most basic choice model and is useful as a base or comparison when estimating more complex models. ${ }^{43}$ However, given that preference heterogeneity may be anticipated around these results, a mixed MNL (MMNL) model will also be used. The MMNL model relaxes some of the more strict assumptions of the MNL model which are unlikely to hold in practice. ${ }^{51}$ One of the main advantages of using an MMNL model is that one or more coefficients can be treated as random and allows the researcher to test if there is significant heterogeneity around average parameter estimates.

There are a number of individual characteristics that will be tested for inclusion in the model which may explain some variation in the results. These include sociodemographic variables for both patients and nurses (age and gender), a number of variables specific to patients (eg, marital status, highest level of education, number of hospitalisations in the previous year) and a number of variables specific to nurses (eg, level of responsibility, workload). Hospital level variables such as whether the hospital is privately or publically funded will also be considered.

\section{ETHICS AND DISSEMINATION}

Ethics approval was sought for both the qualitative analysis and DCE survey from the two hospitals involved as well as a university ethics committee. All participants were, or will be, given participant information sheets and sufficient time to consider this information before agreeing to participant. No incentive payments will be provided to any participants.

A training manual has been developed for the nurse researchers who will collect the main survey data. This manual details how patients and nurses should be approached to ensure all ethical standards are met. It is very important to reassure both patients and nurses that
Figure 1 Example choice set from the patient survey.

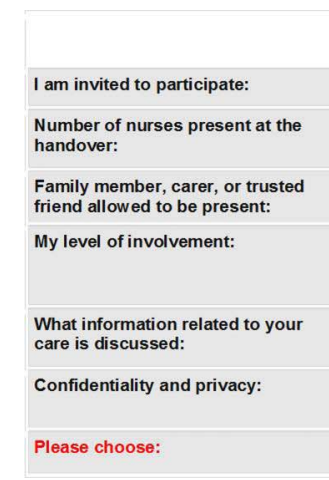


Table 3 Example of choice set seen by patient participant

\begin{tabular}{|c|c|c|c|}
\hline & Handover $\mathrm{A}$ at your bedside & Handover B at your bedside & $\begin{array}{l}\text { I would prefer handover } \\
\text { to happen away from my } \\
\text { bedside }\end{array}$ \\
\hline I am invited to participate & No & Yes & \\
\hline $\begin{array}{l}\text { Number of nurses present at } \\
\text { the handover }\end{array}$ & $\begin{array}{l}\text { Only the nurse leaving and } \\
\text { the nurse coming on }\end{array}$ & $\begin{array}{l}\text { The nursing TEAM leaving and } \\
\text { the TEAM coming on }\end{array}$ & \\
\hline $\begin{array}{l}\text { Family member, carer, or } \\
\text { trusted friend allowed to be } \\
\text { present }\end{array}$ & Yes & No & \\
\hline My level of involvement & $\begin{array}{l}\text { I hear what is said and I am } \\
\text { asked questions }\end{array}$ & $\begin{array}{l}\text { I hear what is said, I am asked } \\
\text { questions and I can speak up } \\
\text { at any time }\end{array}$ & \\
\hline $\begin{array}{l}\text { What information related to } \\
\text { your care is discussed }\end{array}$ & $\begin{array}{l}\text { Information about my medical } \\
\text { condition and plan for care }\end{array}$ & $\begin{array}{l}\text { Information about my medical } \\
\text { condition only }\end{array}$ & \\
\hline Confidentiality and privacy & $\begin{array}{l}\text { Sensitive information is } \\
\text { handed over verbally away } \\
\text { from my bedside }\end{array}$ & $\begin{array}{l}\text { Sensitive information is handed } \\
\text { over in written form }\end{array}$ & \\
\hline Please choose & & & \\
\hline
\end{tabular}

their responses are completely anonymous and cannot be identified from the survey software colleting the data so that patients do not think that any negative feedback may be provided back to the nurses which may affect their immediate care. For nurses, anonymity ensures that they would not 'get into trouble' for holding views or preferences which are contrary to the recommended guideline for bedside handover. It may also help to mitigate any response bias whereby nurses anticipate that managers will want survey results to reflect positively on bedside handover, even if the participant does not share this view. For this reason, once the survey has been completed, participants are told they cannot have their responses removed from the database. Qualitative questions at the end of the survey allow for any concerns to be raised, or to record the reason for non-completion if participants pull-out through the survey.

Results of the main survey will be presented in nursing forums at each participating hospital. Results will be communicated to the funding body by way of an annual report and through published papers. Results will also be disseminated at a number of international quality and safety and nursing conferences.

Importantly, the results of this DCE can inform our three main aims by:

- Identifying, comparing and contrasting how different attributes are perceived by patients and nurses and especially to identify any disconnect. This is important as the two groups are likely to have different, if overlapping, preferences for how handover is conducted in hospitals.

- Identifying any attributes that may be causing nursing staff not to undertake handover in a manner that encourages patient participation in the bedside handover, as per the recommended guidelines. ${ }^{2}$ This is important within a knowledge translation framework ${ }^{52}$ as if we understand the barriers to bedside handover, we can target specific strategies to overcome them and increase the likelihood of uptake.

Author affiliations

${ }^{1}$ Centre for Applied Health Economics, Menzies Health Institute Queensland, Griffith University, Brisbane, Queensland, Australia

${ }^{2}$ National Centre of Research Excellence in Nursing Interventions for Hospitalised Patients, Centre for Health Practice Innovation, Menzies Health Institute Queensland, Griffith University, Brisbane, Queensland, Australia ${ }^{3}$ Faculty of Health, Centre for Quality and Patient Safety, School of Nursing and Midwifery, Deakin University and Alfred Health, Melbourne, Victoria, Australia

${ }^{4}$ Centre for Health Practice Innovation, Menzies Health Institute Queensland, Griffith University, Brisbane, Queensland, Australia

${ }^{5}$ Faculty of Health and Behavioural Sciences, School of Pharmacy, The University of Queensland, Brisbane, Queensland, Australia

Twitter Follow Tracey Bucknall at @Tracey Bucknall@nursedecisions

Contributors WC, TB and JAW wrote the initial grant application. JS wrote the first draft of the protocol. JS, WC, GT, TB and JAW contributed to the drafting and editing of the protocol, and approved the final version.

Funding This work was supported by the Australian Research Council (ARC), Discovery grant number DP130100316.

Competing interests None declared.

Patient consent Obtained.

Ethics approval Griffith University; Gold Coast Hospital; Cabrini Hospital.

Provenance and peer review Not commissioned; externally peer reviewed.

Open Access This is an Open Access article distributed in accordance with the Creative Commons Attribution Non Commercial (CC BY-NC 4.0) license, which permits others to distribute, remix, adapt, build upon this work noncommercially, and license their derivative works on different terms, provided the original work is properly cited and the use is non-commercial. See: http:// creativecommons.org/licenses/by-nc/4.0/

\section{REFERENCES}

1. Australian Commission on Safety and Quality in Healthcare. External evaluation of the National Clinical Handover Initiative Pilot Program. Sydney: ACSHQC, 2011. 
2. Australian Medical Association. Safe handover: Safe patients. Guidelines on clinical handover for clinicans and managers. 2006.

3. Rutherford P, Lee B, Greiner A. Transforming care at the bedside. Cambridge, MA: Institute for Healthcare Innovations, 2004.

4. McMurray A, Chaboyer W, Wallis M, et al. Patients' perspectives of bedside nursing handover. Collegian 2011;18:19-26.

5. Chaboyer W, McMurray A, Wallis M, Bedside nursing handover: a case study. Int $J$ Nurs Pract 2010;16:27-34.

6. Tobiano G, Marshall A, Bucknall T, et al. Patient participation in nursing care on medical wards: an integrative review. Int J Nurs Stud 2015;52:1107-20.

7. Australian Commission on Safety and Quality in Healthcare. Standard 6, Clinical Handover: Safety and Quality Improvement Guide. Sydney, NSW, Australia.

8. Chin G, Warren N, Kornman L, et al. Patients' perceptions of safety and quality of maternity clinical handover. BMC Pregnancy Childbirth, 2011;11:58.

9. Liu W, Manias E, Gerdtz M. Medication communication between nurses and patients during nursing handovers on medical wards: a critical ethnographic study. Int J Nurs Stud 2012;49:941-52.

10. Anderson J, Malone L, Shanahan K, et al. Nursing bedside clinical handover-an integrated review of issues and tools. J Clin Nurs 2015;24:662-71.

11. Riesenberg LA, Leitzsch J, Cunningham JM. Nursing handoffs: a systematic review of the literature. Am J Nurs 2010;110:24-34 quiz 35-6.

12. Staggers N, Blaz JW. Research on nursing handoffs for medical and surgical settings: an integrative review. J Adv Nurs 2013;69:247-62.

13. Australian Commission on Safety and Quality in Healthcare. A structured evidence-based literature review regarding the effectiveness of improvement interventions in clinical handover. Australia: eHealth Services Research Group, University of Tasmania, 2008.

14. Kerr D, Lu S, McKinlay L, et al. Examination of current handover practice: evidence to support changing the ritual. Int $J$ Nurs Pract 2011;17:342-50.

15. McMurray A, Chaboyer W, Wallis M, et al. Implementing bedside handover: strategies for change management. $J$ Clin Nurs 2010;19:2580-9.

16. Evans AM, Pereira DA, Parker JM. Discourses of anxiety in nursing practice: a psychoanalytic case study of the change-of-shift handover ritual. Nurs Inq 2008;15:40-8.

17. Chaboyer W, McMurray A, Johnson J. Bedside handover: quality improvement strategy to "transform care at the bedside". J Nurs Care Qual 2009;24:136-42.

18. Taylor C. Assessing patients' needs: does the same information guide expert and novice nurses? Int Nurs Rev 2002;49:11-19.

19. Scovell S. Role of the nurse-to-nurse handover in patient care. Nurs Stand 2010;24:35-9.

20. Kerr D, Lu S, McKinlay L, Towards patient-centred care: perspectives of nurses and midwives regarding shift-to-shift bedside handover. Int J Nurs Pract 2014;20:250-7.

21. Wilson R. Improving clinical handover in emergency departments. Emerg Nurse 2011;19:22-6.

22. O'Connell B, Penney W, Challenging the handover ritual. Collegian 2001;8:14-18.

23. Anderson C, Mangino R. Nurse shift report: who says you can't talk in front of the patient? Nurs Adm Q 2006;30:112.

24. Johnson M, Cowin LS. Nurses discuss bedside handover and using written handover sheets. J Nurs Manag 2013;21:121-9.

25. Jeffs $L$, Cardoso R, Beswick $S$, et al. Enablers and barriers to implementing bedside reporting: insights from nurses. Nurs Leadersh 2013;26:39-52.

26. Jeffs $L$, Acott A, Simpson E, et al. The value of bedside shift reporting enhancing nurse surveillance, accountability, and patient safety. J Nurs Care Qual 2013;28:226-32.
27. Drach-Zahavy A, Shilman O. Patients' participation during a nursing handover: the role of handover characteristics and patients' personal traits. J Adv Nurs 2015;71:136-47.

28. Timonen L, Sihvonen M. Patient participation in bedside reporting on surgical wards. J Clin Nurs 2000:9:542-8.

29. Johnson M, Jefferies D, Nicholls D. Exploring the structure and organization of information within nursing clinical handovers. Int $J$ Nurs Pract 2012;18:462-70.

30. Cahill J. Patient's perceptions of bedside handovers. J Clin Nurs 1998;7:351-9.

31. Lu S, Kerr D, McKinlay L. Bedside nursing handover: patients' opinions. Int J Nurs Pract 2014;20:451-9.

32. Jeffs L, Beswick S, Acott A, et al. Patients' views on bedside nursing handover: creating a space to connect. J Nurs Care Qual 2014;29:149-54.

33. Greaves C. Patients' perceptions of bedside handover. Nurs Stand 1999;14:32-5.

34. Kerr D, McKay K, Klim S, et al. Attitudes of emergency department patients about handover at the bedside. J Clin Nurs 2014;23:1685-93.

35. de Bekker-Grob EW, Ryan M, Gerard K. Discrete choice experiments in health economics: a review of the literature. Health Econ 2012;21:145-72.

36. Ryan M. Discrete choice experiments in health care. $B M J$ 2004;328:360-1.

37. Thurstone LL. A law of comparative judgment. Psychol Rev 1994:101:266-70.

38. Lancaster K. A new approach to consumer theory. J Polit Econ 1966;74:132-57.

39. Bridges JFP, Hauber AB, Marshall D, et al. Conjoint analysis applications in health-a checklist: a report of the ISPOR good research practices for conjoint analysis task force. Value Health 2011;14:403-13.

40. Coast J, Al-Janabi $\mathrm{H}$, Sutton EJ, et al. Using qualitative methods for attribute development for discrete choice experiments: issues and recommendations. Health Econ 2012;21:730-41.

41. Tobiano G, Bucknall T, Marshall A, et al. Patients' perceptions of participation in nursing care on medical wards. Scand J Caring Sci 2015 [epub ahead of print 2 Jun 2015]. doi:10.1111/scs.12237.

42. Tobiano G, Bucknall T, Marshall A, et al. Nurses' perceptions of patient participation in nursing care. J Adv Nurs 2015 [epub ahead of print 2015]. doi: 10.1111/jan.12740.

43. Hensher DA, Rose JM, Green WH. Applied choice analysis: a primer. Cambridge University Press, 2005.

44. Whitty JAP, Carrington MJ, Stewart S, et al. Patient preferences for the delivery of disease management in chronic heart failure: a qualitative study. J Cardiovasc Nurs 2012;27: 201-7.

45. Reed Johnson F, Lancsar E, Marshall D, et al. Constructing experimental designs for discrete-choice experiments: report of the ISPOR conjoint analysis experimental design good research practices task force. Value Health 2013;16:3-13.

46. ChoiceMetrics, Ngene 1.1.2. 2014

47. ChoiceMetrics, Ngene User Manual and Reference Guide. 2014

48. Rose JM, Bliemer MCJ. Constructing efficient stated choice experimental designs. Transport Rev 2009;29:587-617.

49. de Bekker-Grob EW, Donkers B, Jonker MF, et al., Sample size requirements for discrete-choice experiments in healthcare: a practical guide. Patient 2015;8:373-84.

50. Johnson R, Orme B. Getting the most from CBC. Sequim: Sawtooth Software Research Paper Series, Sawtooth Software. 2003.

51. Train K. Discrete choice methods with simulation. 2nd edn Cambridge University Press, 2009.

52. Grol R, Grimshaw J. From best evidence to best practice: effective implementation of change in patients' care. Lancet 2003;362:1225-30. 